Meta

Journal des traducteurs

Translators' Journal

\title{
Index of Words and Topics in Volume 14
}

Volume 14, numéro 4, décembre 1969

URI : https://id.erudit.org/iderudit/003195ar

DOI : https://doi.org/10.7202/003195ar

Aller au sommaire du numéro

Éditeur(s)

Les Presses de l'Université de Montréal

ISSN

0026-0452 (imprimé)

1492-1421 (numérique)

Découvrir la revue

Citer ce document

(1969). Index of Words and Topics in Volume 14. Meta, 14(4), 268-271.

https://doi.org/10.7202/003195ar d'utilisation que vous pouvez consulter en ligne.

https://apropos.erudit.org/fr/usagers/politique-dutilisation/ 


\section{INDEX OF WORDS AND TOPICS IN VOLUME 14}

A la mode, 3, p. 150

Abbreviations, 1, p. 59 .

Abovementioned, 3, p. 144.

Absolute assignment, 4, p. 245.

Abstract (in French), 4, p. 197.

Acceptance, 4, p. 210.

Acceptance test, 4, p. 210

Acronyms, 1, p. 59 .

Action, 1, p. 43 .

Active to passive voice, 3, p. 148.

Adding machine, 2, p. 113

Advanced research, 3, p. 147.

Adverbial particles, 2, p. 118.

Aforesaid, 3, p. 144

Air conditioning, 2, p. 118

Air turbulence, 3 , p. 143.

All things to all men, 1, p. 23.

Alliteration, 1, p. 24

Amendments, 4, p. 246.

Anchor, 1, p. 37.

And/or, 1, p. 71 .

Angular, 3, p. 139.

Anti-creep device, 1, p. 37

Antimacassar, 1, p. 57.

Antonyms, 1, p. 58

Arabic, 4, p. 249.

Article, 3, p. 142.

Askarel, 4, p. 209

Assignment, 4, p. 245.

Assistant librarian, 2, p. 113.

Assonance, 1, p. 24

At a dash, 3, p. 145

At the top, 3, p. 144.

Atmosphere, 4, p. 195.

Automated express terminal, 3, p. 161

Automatic translation, 2, p. 119.

Auto-transformer, 4, p. 209.

Auto-transporter, 1, p. 36.

Bad translation, 1, p. 30.

Baked, 3, p. 143.

Ballasting, 1, p. 37

Base of rail, 1, p. 37

Basis (on the b...), 3, p. 156.

Be harsh, 4, p. 197

$\mathrm{Be}+$-ing, 1, p. 52

Benefit, 4, p. 245

Bible translating, 3 , p. 165

Bi-directional, 1, p. 36

Bi-level auto-transporter, 1, p. 36.

Blind flying, 2, p. 94.

Blood count, 2, p. 94

Blue sky laws, 2, p. 105.

Board of governors, 3, p. 159.

Body corporate and politic, 2, p. 97.

Book lover, 2, p. 94

Boom, 2, p. 94

Boom, 2, p. 101

Break, 2, p. 100

Breast, 4, p. 199

Breather, 4, p. 209.

Breeder, 2, p. 94.

Brief, 3, p. 162
Bring (to) someone from the outside, 3, p. 150.

Budget, 2, p. 113.

Bugging devide, 2, p. 102.

Bulıdozer, 3, p. 179

Bushing, 4, p. 209.

"Café ", 2, p. 107.

Cafeteria, 2, p. 110.

Cake-walk, 2, p. 110

Call (to) in the law, 3, p. 147

Canadianisms, 2, p. 117

Capacitance, 4, p. 209

Car-ferry, 2, p. 110

Carter, 2, p. 110

Cash flow, 2, p. 104.

Cashier, 2, p. 113.

Catenary, 1 , p. 35

Cause-effect relationship, 3, p. 147

Charitable corporation, 1, p. 46

Chest, 4, p. 199

Claim, 4, p. 245.

Climatic word, 3, p. 151

Coach, 1, p. 35 .

Collateral assignment, 4, p. 245.

Color, 4 , p. 217.

Come (to) up through the ranks, 3, p. 150 .

Comfort and convenience, 2, p. 97.

Commercial laundry, 4, p. 212.

Commercial terms, 2, p. 115.

Commission, 3, p. 159

Committee, 3, p. 159.

Communication, 3, p. 145.

Company, 1, p. 46.

Comparative particles, 2, p. 122.

Conference, 4 , p. 239

Conference interpreting, 2, p. 119.

Connotative meaning, 3 , p. 166.

Consideration (in... of ), 3, p. 156.

Container, 3, p. 160.

Contrastive, 1, p. 13.

Control room, 2, p. 102.

Conversion privilege, 4, p. 247.

Cooling fan, 4, p. 210.

Core type transformer, 4, p. 209.

Cornish names, 4, p. 248.

Corporation, 1, p. 46.

Cosmetology, 4, p. 243

Cottage cheese, 4, p. 219.

Cover, 4, p. 210

Crane, 2, p. 101

Crumble (to), 3, p. 150.

Crystal set, 3, p. 147.

Cuisine, $3, p .150$

Current collector, 1 , p. 35

Current transformer, 4, p. 211.

Cut away (to) dead wood, 2, p. 97

Czech language, 2, p. 117.

Data processing system, 1, p. 38.

Date stamp, 2, p. 113

Deadheading, 1, p. 35 .

Deceptive cognates, 3 , p. 136

Decision-making, 3, p. 151.

Decision-taking, 3, p. 151 . 
Dehydrator, 4, p. 209.

Del credere agent, 2, p. 113

Delco, 2, p. 110.

Delta connection, 4, p. 210.

Demand, 1, p. 43.

Designer, 3, p. 179.

Destroy (to), 3, p. 142.

Dialect, 4, p. 250

Diesel, 1, p. 35

Disability income plan, 3, p. 169

Diverter switch, 4 , p. 210 .

Dividend, 4, p. 245

Divisible surplus, 4, p. 246.

Do not fold, 3, p. 136 .

Dodg'ems, 3, p. 146.

Dog show, 2, p. 94

Door price, 2, p. 94.

Driving mechanism, 4, p. 210

Dry-type transformer, 4 , p. 209.

Durative aspect, 3 , p. 150

Edit (to), 4, p. 207.

Edit (to) a film, 4, p. 207

Editing, 4, p. 207-208.

Edition, 4, p. 208.

Editor, 4, p. 207.

Editorial, 4, p. 207-208.

Editorial writer, 4, p. 208

Electrify (to), 3, p. 139.

Electronics, 1, p. 59

Employee, 3, p. 154

Employee pension fund, 3, p. 156.

Employee's contribution, 3, p. 156

Employer's contribution, 3, p. 156.

Endorsement, 4, p. 245.

Engineering shop, 2 , p. 97

English linguistics, 3, p. 172.

English mind, 3, p. 143.

English predicate, 4, p. 249

English river names, 4, p. 248.

English stylistics, 1, p. 57

English translation, 3, p. 141

Environment, 1, p. 37.

Epigrams, 1, p. 56

Every time, 3 ,p. 149.

Executive office, 2, p. 114.

Expansion tank, 4, p. 209.

Explosion vent, 4, p. 209.

Explosion vent pipe, 4 , p. 210.

Expressionistic translation, 4, p. 196

Facilities, 1, p. 36

Fade (to), 3, p. 145

Feature, 3, p. 138

Feed (to), 2 , p. 113

Fidelity, 1, p. 23, 29

Film editing, 4 , p. 208

Financial terms, 2, p. 115.

Fine and dandy, 2, p. 97.

Flag waving, 2, p. 94.

Flange, 1, p. 37

Flanged wheels, 1, p. 34

Flat car, 1, p. 36

Flat car with stakes, 1, p. 36

Flat car with wraparound bulkheads, 1, p. 36.

Flounder (to), 3, p. 139.

Folk-etymology, 4, p. 247

Fondu(e) , 4, p. 220 .

Foreign words and phrases, 1, p. 58

Freedom, 1, p. 25.
Free-wheeling transiation, 4, p. 196.

Freight car, 1, p. 35 .

Freight equipment, 1, p. 35.

French mind, 3, p. 143.

French vocabulary, 2, p. 118.

French-Canadian Verse, 1, p. 27.

From earliest infancy, 3, p. 147.

Fuel, 2, p. 110.

Gazoil, 2, p. 110.

Genetics, 1, p. 59

Giraffe, 2, p. 101.

Gondola car, 1, p. 36.

Goodwill, 4, p. 218.

Grammatical order of words, 2, p. 122

Great picture, 3, p. 138

Grop (to) one's way around, 3, p. 146

Handle (to) a sword, 3, p. 142 .

Hardness, 4, p. 197.

Hardware, 2, p. 101, 103

Harmonic value of a poem, 4, p. 195.

Harshness, 4, p. 197.

Hawaiian, 3 , p. 170 .

Head, 3, p. 166.

Head of rail, 1, p. 37.

Heart, 4, p. 199.

Hire (to) away, 3, p. 150.

Hopper car, 1, p. 36.

Hospital linen, 4, p. 217

Human interest stories, 1, p. 56

Humor, 2, p. 119

Ideal translator, 1, p. 23.

Image, 4, p. 196.

Immersed, 4, p. 209.

Implicitation, 3, p. 143, 145.

Impulse-voltage withstand test, 4, p. 210.

Indiscriminate admiration, 2 , p. 97 .

Inerteen, 4, p. 209.

Inhalatory therapy, 1, p. 78 .

Input, 4, p. 242.

Institutional laundry, 4 , p. 210.

Instrument transformer, 4, p. 211.

Insulated car, 1, p. 36.

Insulating liquid, 4, p. 209

Intellectual value of a poem, 4, p. 195

Interconnected star connection, 4, p. 210.

Intercontinental, 3, p. 161

Intermodal, 3, p. 161

Intracontinental, 3, p. 161

Issue (to), 4, p. 245.

Interative aspect, 3 , p. 150

Jamaican English, 2, p. 123.

Jingles, 3, p. 180.

Jitney, 2, p. 104.

Jump-rope rhymes, 3, p. 170.

Kinescope, 2, p. 113

Kurdish, 1, p. 58.

Labour turnover, 3, p. 169.

Ladle-car, 1, p. 36.

Laundry, 4, p. 211.

Leader, 2, p. 110.

Leadership, 2, p. 110 .

Leakage, 1, p. 71

Lecture course, 3 , p. 138

Let (to) the wind blow in, 3, p. 142 .

Let us be hard, 4, p. 197.

Lexical field, 4, p. 204.

Line-up (editor), 2, p. 113.

Literal poetic, 4, p. 196. 
Literary translation, 1 , p. 3-33; 4, p. 249

Live (to) on the wrong side of the tracks, 3 , p. 137.

Load loss, 4, p. 210.

Logical circuits, 1, p. 37.

Long drinks, 2, p. 110.

Loss, 4 , p. 210.

Lower (to), 4, p. 210.

Machine tool, 1, p. 60.

Machine translation, 3, p. 171.

Magnetic core, 4, p. 209.

Management, 1, p. 59;2, p. 102; 4, p. 211.

Market forecasting, 2, p. 118.

Marketing, 2, p. 102.

Mason-Dixon Line, 1, p. 57

Masterly course, 3 , p. 138

Material witness, 1, p. 44.

May be open for inspection, 1, p. 10.

Meaning, 4, p. 195.

Measurement of impedance voltages, 4, p. 210.

Measures, 1, p. 59.

Mechanical refrigerator, 1 , p. 36

Mechanics, 3, p. 171.

Meeting (the) will come to order, 3, p. 136.

Melt (to) down, 3, p. 139

Men at work, 3, p. 144.

Metering outfit, 4, p. 211

Metering unit, 4, p. 211.

Mineral oil, 4, p. 209

Mixing console, 2, p. 102 .

Mixing panel, 2, p. 102 .

Moderator, 3, p. 146.

Modulation, 3, p. 146

Monitoring desk, 2, p. 102.

More (two hundred or...), 3, p. 149.

Morphology, 4, p. 249.

Motive power, 1 , p. 35 .

Motors, 3, p. 147.

Musical, 3, p. 139.

National dialogue, 4, p. 195

Native language, 3, p. 143, 145.

Nature of translating, 3 , p. 165.

Nautical termonology, 1, p. 59 .

Need of translation, 4, p. 195.

Negation, 2, p. 122; 4, p. 197.

Negative pledge, 2, p. 105.

News editing, 4, p. 208.

Nine out of ten, 3 , p. 152 .

No action will be dismissed, 1, p. 44.

No dumping, 1, p. 10.

No load loss, 4, p. 210.

No parking at any time, 2, p. 97

Nominee, 2, p. 104.

Non-mechanical refrigerator, 1 , p. 36.

Nucleonics, 1, p. 59.

Object (the) of the demand, 1, p. 43

Occupational therapist, 2, p. 101.

Officer, 3, p. 155.

On stop (bourse), 2, p. 105.

On-load tap changer, 4, p. 210.

Operated, 4, p. 210.

Organic compounds, 1, p. 59.

Organic flow of a poem, 4, p. 196.

Organization pattern, 2, p. 97.

Overhead contact line, 1, p. 35.

Paddle (to), 3, p. 139.

Pads, 4, p. 251.

Pantograph, 1, p. 35.
Paper clips, 2, p. 114.

Participation in profits, 4, p. 245 .

Passenger car, 1, p. 35 .

Passenger equipment, 1, p. 35

Pattern, 3, p. 164

Patterns of thought, 3, p. 141

Perfective aspect, 3, p. 150.

Petits fours, 3 , p. 150 .

Phonology, 4, p. 249.

Piggyback trailer, 1, p. 36.

Plane of abstraction, 3, p. 141.

Plane of reality, 3, p. 141

Plug-door car, 1, p. 36.

Plus tick, 2, p. 106.

Poetic free-wheeling translation, 4, p. 196.

Poetic universe, 4, p. 195.

Poetry, 1, p. 27; 4, p. 196.

Poetry and translation, 4, p. 249.

Policy change, 4, p. 246.

Policy year, 4, p. 244.

Policyowner's dividend, 4, p. 245.

Polytechnical dictionary, 4, p. 248.

Postal inspection (may be open for), 1, p. 10.

Potential transformer, 4, p. 211.

Power dome car, 1 , p. 36 .

Power transformer, 4, p. 209

Practice of translation, 3, p. 165.

Precision, 1, p. 25.

Preposition, 2, p. 118.

Present participle, 1, p. 25.

Primary, 4, p. 210.

Procedure, 1, p. 37

Process cheese, 4, p. 219.

Processed cheese, 4, p. 219.

Profession (definition), 2, p. 89

Professional status, 2, p. 89

Pronounciation, 1, p. 58.

Proverbs, 1, p. 56.

Publisher, 4, p. 207.

Pullover, 2, p. 110

Pump, 4, p. 210.

Punctual aspect, 3, p. 150.

Put through, 2, p. 105.

Radiation chemistry, 4, p. 232.

Radioactive nuclides, 1, p. 60.

Radio-chemistry, 4, p. 232.

Railroad, 1, p. 34; 2, p. 116.

Railway, 2, p. 116

Railway crossing, 1, p. 37.

Raise (to), 4, p. 210.

Rated capacity, 4, p. 209.

Rated power, 4, p. 209.

Ratio adjuster, 4, p. 210.

Recognition, 2, p. 91.

Reel-car, 1, p. 36.

Reels, 3, p. 147.

Referential meaning, 3, p. 166.

Rejection, 4, p. 210.

Relative pronoun, 4, p. 198.

Remake, 2, p. 110.

Resound (to), 3, p. 142.

Restructuring, 3, p. 166

Retirement income plan, 3, p. 169.

Rewrite, 2, p. 110.

Rhetorical terms, 1, p. 60.

Road bed, 1, p. 36 .

Roll-on roll off, 3, p. 161.

Routine test, 4, p. 210. 
Rules and regulations, 2, p. 97.

Rhythm, 4, p. 196.

Safety features, 3, p. 143.

Said, 3, p. 143.

Sale forecasting, 2, p. 118.

Scarecrow, 4, p. 197.

Scientific translation, 1, p. 58.

Seating capacity, 3 , p. 148.

Secondary, 4 , p. 210.

Self-cooled, 4, p. 210.

Self-service, 2, p. 110.

Sew (to) on, 3, p. 139

Shade, 3, p. 179.

Shareholder dividend, 4, p. 245.

Shatter (to), 3, p. 142.

Shell type transformer, 4, p. 209.

Shortening, 4, p. 220.

Silica-gel, 4, p. 209.

Sleeping-car, 1, p. 35.

Slosh (to) around, 3, p. 139.

Soap, 4, p. 215.

Software, 2, p. 101, 103.

Solid, 3, p. 143.

Sound effects, 2, p. 102.

Sound value, 4, p. 196.

Source language, 1, p. 5.

Spanish, 1, p. 58,60 .

Special effects, 2, p. 102.

Specification, 3, p. 143, 145.

Speech closers, 1, p. 56.

Speech openers, 1, p. 56.

Spread cheese, 4, p. 222.

Standard Average European, 4, p. 203.

Star connection, 4, p. 210.

Streamlined, 2, p. 93.

Stress, 1, p. 24

Strictly prohibited, 3, p. 136.

Structural translation, 4 , p. 248 .

Style and stylistics, 1, p. 57.

Styles of translation, 4, p. 196.

Stylistic context, 3, p. 164.

Stylistic device, 3 , p. 163.

Substitution, 1, p. 23.

Sums of money, 3, p. 147.

Sunbath, 2, p. 114.

Supervisory staff, 4, p. 211.

Support (to), 2, p. 97

Surnames, 4, p. 248.

Surplus earnings, 4, p. 246

Switch (to), 2, p. 105 .

Switching, 2, p. 105

Synecdoche, 3 , p. 147.

Synonyms, 1, p. 58; 2, p. 117.

Syntex, 4, p. 196.

Table microphone, 2, p. 102.

Table radio, 2, p. 102.

Table record player, 2 , p. 102.

Table tripod, 2, p. 102 .

Table set, 2, p. 102 .

Table TV set, 2, p. 102.

Tail gate leader, 3 , p. 162.

Tank, 4, p. 209.

Tap, 4, p. 210.

Tap (to), 2, p. 102.

Target language, 1, p. 5 .

Telecommunication, 1, p. 59.

Telewriter, 2, p. 113

Temperature controlled equipment, 3, p. 161.

Termina], 4, p. 209.
Terms and conditions, 2, p. 97.

Tertiary, 4, p. 210.

Test, 4, p. 210.

Test chart, 4, p. 243.

Test of temperature rise, 4 , p. 210

Theory of translation, 3, p. 165 .

There is no such thing as perfection, 3, p. 137.

There is nothing like, 3, p. 137.

Thief-proof system, 3 , p. 143 .

Throbbing, 3, p. 142.

Ticker, 2, p. 105.

Tie laying, 1, p. 37.

Tint, 3, p. 179.

Tone, 3, p. 179.

Tonic, 1, p. 24.

Trade name creation, 4, p. 249.

Trade-in allowance, 2, p. 97.

Trader, 2, p. 105.

Trading, 2, p. 105

Trailer, 2, p. 113.

Training ground, 3, p. 146

Transcontainer, 3, p. 161

Transfer, 3, p. 166.

Transference of feeling, 4, p. 198

Transformulation, 4, p. 248.

Translation, 3, p. 171

Translation limit, 2, p. 119

Translation machine, 1, p. 23.

Translation of poetry, 1, p. 30; 4, p. 195

Translator-client relationship, 2, p. 90.

Tri-level auto-transporter, 1 , p. 36.

Turn, 4, p. 210.

Turn-table, 2, p. 102.

Two-door layout, 3 , p. 141.

Two-way track, 1, p. 37.

Type test, 4, p. 210.

Typhoid infection, 3, p. 144

Underworld (dictionary of the) , 4, p. 247.

Up tick, 2, p. 106.

Vague, 4, p. 197.

Vectorial indications, 3, p. 151.

Verb system, 1, p. 24.

Video recording, 2, p. 113.

Vocabulary, 4, p. 196.

Voltage ratio, 4, p. 210.

Voltage ratio measurement, 4, p. 210.

Voltage transformer, 4, p. 211.

Voting trust, 2, p. 106.

Wage incentive plan, 3, p. 169.

Walk (to), 3 , p. 139 .

Washer, 4, p. 214

Water, 4, p. 216

Ways and means, 2, p. 97.

We must be hard, 4, p. 197

Web of the rail, 1, p. 37.

Weights and measures, i, p. 59

Wheels, 3, p. 147

Whenever, 3, p. 149

Winding, 4, p. 209.

Wire tap (to), 2, p. 102.

Witness, 1 , p. 44 .

Work force, 4, p. 211.

Worn away / out, 3, p. 139

Yao, 3, p. 170.

Yard engine, 1, p. 35.

You are telling me, 3 , p. 137

Zigzag connection, 4, p. 210.

Zip code, 2, p. 114 .

Zoom, 2, p. 110. 